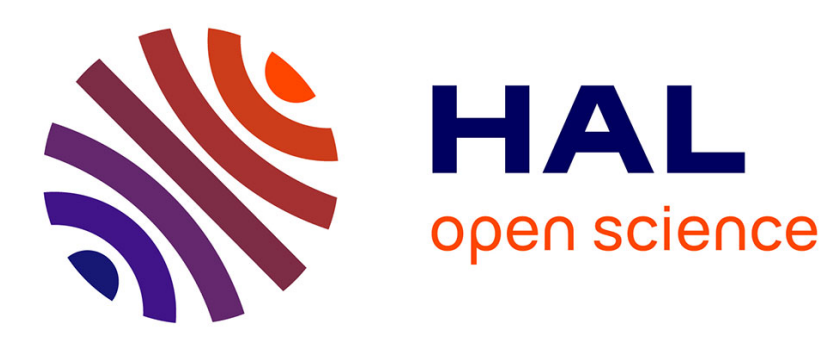

\title{
A linguistic approach for non-functional constraints in a semantic SOA environment
}

Pierre Châtel, Isis Truck, Jacques Malenfant

\section{To cite this version:}

Pierre Châtel, Isis Truck, Jacques Malenfant. A linguistic approach for non-functional constraints in a semantic SOA environment. FLINS 2008 - 8th International FLINS Conference on Computational Intelligence in Decision and Control, Sep 2008, Madrid, Spain. pp.889-894, 10.1142/9789812799470_0146. hal-01243541

\section{HAL Id: hal-01243541 \\ https://hal.science/hal-01243541}

Submitted on 15 Dec 2015

HAL is a multi-disciplinary open access archive for the deposit and dissemination of scientific research documents, whether they are published or not. The documents may come from teaching and research institutions in France or abroad, or from public or private research centers.
L'archive ouverte pluridisciplinaire $\mathbf{H A L}$, est destinée au dépôt et à la diffusion de documents scientifiques de niveau recherche, publiés ou non, émanant des établissements d'enseignement et de recherche français ou étrangers, des laboratoires publics ou privés. 


\title{
A linguistic approach for non-functional constraints in a semantic SOA environment
}

\author{
Pierre CHÂTEL \\ Thales Communications France \\ 1-5 avenue Carnot, Massy, 91883, France \\ E-mail: pierre.chatel@fr.thalesgroup.com \\ Isis TRUCK \\ LIASD, University Paris 8 \\ 2 rue de la Liberté, Saint-Denis Cedex, 93526, France \\ E-mail: truck@ai.univ-paris8.fr \\ Jacques MALENFANT \\ Université Pierre et Marie Curie-Paris 6, CNRS, UMR 7606 LIP6 \\ 104 av. du Président Kennedy, Paris, 75016, France \\ E-mail: Jacques.Malenfant@lip6.fr
}

\begin{abstract}
This contribution addresses the problem of expressing preferences among nonfunctional properties in a Web Service architecture. In such a context, semantic annotations are needed and added on service declaration and business process in order to select the best available service. These conditional and unconditional preferences are managed using Conditional Preference-Networks (CP-Nets). But in several cases, uncertainty related to the preferences has to be taken into account to achieve a better satisfaction rate. We propose the use of fuzzy linguistic information inside the whole process when it will be necessary.
\end{abstract}

Keywords: preference modelling, fuzzy linguistic approach, CP-Nets, Web service filtering

\section{Introduction}

Service-oriented architectures (SOA) deal with the growing need for distributed applications capable of evolving continuously over their execution. In the context of the web, services can appear and disappear at runtime, thus requiring a loose coupling between service providers and consumers. To get this loose coupling, we adopt the late-binding of calls to services, by deciding at runtime which service will be called to satisfy each con- 
sumers' request. We use consumers' preferences over the functional and non-functional characteristics to choose among candidate services.

We concur with Schröpfer et al. ${ }^{11}$ to use a CP-Nets approach to elicit and exploit consumers preferences in a Semantic Service Oriented Architecture (SSOA). But we use the fuzzy linguistic approach ${ }^{12}$ to model nonfunctional preferences for the dynamic selection of Web services. These preferences express complex requirements, defined inside Web processes, that are related to the different non-functional characteristics of the services that will be needed to guarantee the application's execution (an SOA application is a set of Web processes and dynamically discovered Web services).

In the rest of the paper, we first introduce our technical framework, then we briefly describe "*CP-Nets"*. Section 4 introduces our proposal of using fuzzy linguistic in modeling preferences, Section 5 provides a proofof-concept example, while Section 6 points out conclusions and future work.

\section{Technical Framework}

\subsection{SOA and Web Services}

In the SOA framework, two distinct roles are identified. Service providers implement (generic) functionalities made available to applications, thanks to SOA standards like, e.g., service directories, as Web services. Service consumers request and use services available on the network according to their specific requirements through service calls made by Web processes.

To cope with the dynamism of the Web, the binding of Web services (from providers) to Web processes (of consumers) is established on the fly, at runtime. To achieve this, and to provide for high interoperability among heterogeneous service offers and requests, we make this binding go much farther than the traditional syntactic approach by using semantic annotations on service offers and requests to identify offers that match each request. In our approach, semantics encompasses both functional (what services do and processes need) and non-functional properties (QoS and related properties guaranteed by Web services and required by Web processes). *"*CP-Nets" means any kind of Conditional Preference Networks, i.e. CP-Nets, TCP-
Nets, UCP-Nets, etc. 


\subsection{Functional and Non-Functional Constraints}

Functionality of service offers concerns the core business work provided by each Web services. We strive to semantically describe the main features of the service: its end goal and the ontological concepts associated to its input and output parameters. We use SAWSDL ${ }^{10}$ to annotate otherwise syntax-based service offers with classes (i.e. concepts) from domain ontologies. Service requests express similarly their semantic requirements for the completion of their task.

But we also strive to build Quality of Service (QoS) awareness into the above SSOA platform to select at runtime the best service(s) available to fulfill each request. Indeed, after filtering with functional constraints, we also use non-functional information to further seek the best service(s). Non-functional requirements are constraints and preferences applied to both statically published and instantaneously measured QoS values associated to the functionally filtered services.

A major issue when dealing with QoS is the large number of different dimensions (e.g. latency, precision, etc.) of importance. Because one rarely gets an offer that is the best for every different QoS dimension, we need consumer preferences to rank offers given their relative strength on the different dimensions. To this end, we propose a new formalism based on the combination of CP-Nets ${ }^{1}$ and the fuzzy linguistic approach ${ }^{12}$ to specify the preferences of web processes over the different QoS properties of offers.

\section{Modelling Preferences Through ${ }^{*}$ CP-Nets}

\subsection{CP-Nets}

A CP-Net (Conditional Preference-Network) is a compact graphical representation of qualitative user preferences. ${ }^{3}$ It is relatively intuitive. Its main elements are: nodes representing the problem variables (properties), arcs denoting preferences among these variables for given values, and the "Conditional Preference Tables" or CPT. CPTs express the preference over values taken by nodes, using binary relationship between them.

CP-Nets allow for the preference modeling of statements such as "I prefer the $V_{1}$ value for property $X$ over $V_{2}$ if properties $Y$ equals $V_{Y}$ and $Z$ equals $V_{Z}$ ". In fact, this graphical representation allows us to express the dependency between connected CPTs. There is also a notion of relative preference between the preferences themselves: a CPT associated with a specific node has a higher priority than the CPTs of its offspring. 


\subsection{Common CP-Nets variants}

Utility CP-Nets, or UCP-Nets, differ from CP-Nets by replacing the binary relationship between node values in CPTs by utility factors. ${ }^{2}$ A utility factor is a real number associated to the value assigned to a node $X$ from the network, given a specific assignment to its parent nodes $\operatorname{Pa}(X)$. Utility factors express preference degrees for the different assignments.

Another extension, named Tradeoffs-enhanced CP-Nets, or TCP-Nets, allows one to express preferences of the form: "A better assignment for $X$ is more important than a better assignment for $Y$ ". These are called relative importance statements. ${ }^{4}$ Using a new kind of preference tables and a new specific arc, TCP-Nets generalize this class of preferences in order to accept conditional relative importance statements. With these, it becomes possible to express preferences of the form: "A better assignment for $X$ is more important than a better assignment for $Y$ given that $Z=z$ ".

Our idea to use the TCP- and UCP-Nets formalisms for service selection in an SOA framework has already been proposed ${ }^{11}$ but we go further by following a fuzzy linguistic approach alongside ${ }^{*} \mathrm{CP}-\mathrm{Nets}$.

\section{Fuzzy Linguistic Approach and CP-Nets}

*CP-Nets exhibit two important limitations to express preferences in a QoS settings. Many QoS dimensions are defined by continuous domain variables, but ${ }^{*} \mathrm{CP}-N e t s$ do not handle continuous variables. We propose to discretize continuous domains using fuzzy linguistic terms ${ }^{12}$ instead of crisp subsets. Another problem is that precise utility values, such as those used in UCPNets, are hard to get from the customers. We propose to express the utility factors with qualitative data, i.e. words translated into fuzzy subsets, later on represented as fuzzy 2 -tuples ${ }^{8}$ to compute global utility functions.

\subsection{Fuzzy Linguistic Approach}

The fuzzy linguistic approach represents qualitative aspects as linguistic values by means of linguistic variables. ${ }^{12}$ Appropriate linguistic descriptors must be chosen to form the term set as well as their semantics. The universe of the discourse over which the term set is defined can be arbitrary. In this paper, we shall use the interval $[0,1]$. Odd cardinality term sets, typically 7 or 9 , are preferred, ${ }^{6,8}$ representing the mid term by an assessment of "approximately 0.5 ", other terms being placed symmetrically around it. For example, a set of five terms $S$, could be given as: $S=\left\{s_{0}:\right.$ very low, $s_{1}$ : low, $s_{2}$ : medium, $s_{3}$ : high, $s_{4}$ : very high\}. 


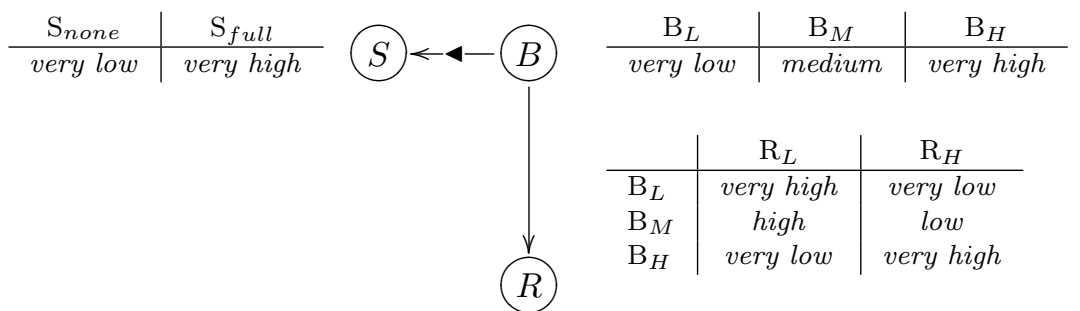

Fig. 1. Imaging web service QoS preferences example using LCP-Nets.

It is also required that there exist negation $N e g$, a $\max$ and $\min$ operators defined over this set. ${ }^{8}$ The use of linguistic variables implies the processes of computing with words for their fusion, aggregation, comparison, etc. To perform these computations, different models have been used such as the semantic, ${ }^{5}$ the symbolic ${ }^{6}$ or the 2 -tuple ${ }^{8}$ representation models.

\subsection{Linguistic CP-Nets (LCP-Nets)}

We propose a new variant of CP-Nets, called LCP-Nets, to get the advantage of the fuzzy linguistic approach into a marriage of UCP-Nets and TCP-Nets. LCP-Nets have nodes corresponding to problem variables which continuous domains are discretized as linguistic term sets. LCP-Nets allow users to express tradeoffs among variables using i-arcs from TCP-Nets. Finally, LCP-Nets have CPTs similar to the ones of UCP-Nets, but express utilities with linguistic terms rather than numerical values.

Those linguistic terms are represented as 2-tuples to allow for the computation of the aggregated global utility function of the LCP-Net from its different CPTs. To perform this aggregation, we use a weighted average operator where the weights are in $[0,1]$, sum to 1 and are decreasing from more important variables (high in the LCP-Net) to less important ones.

\section{Example}

Consider an imaging web service which QoS is defined using three dimensions: security (S), bandwidth (B) and image resolution (R). The user always prefers bandwidth over security, and if the bandwidth is low, he prefers low-resolution images because he simply needs images as fast as possible. Figure 1 gives the corresponding LCP-Net.

Security can be either none or full, given utilities very low and very high respectively. The preference of bandwidth over security is accounted 
for by an i-arc from B to S (an arc with a middle triangle). The bandwidth is discretized using three linguistic variables $B_{L}$ (low), $B_{M}$ (medium) and $B_{H}$ (high). Preferences among these values are given by the CPT beside $\mathrm{B}$, expressing a very low preference for a low bandwidth, a medium one for the medium bandwidth and a very high one for a high bandwidth.

Image resolution is also discretized using two linguistic terms: $R_{L}$ (low) and $R_{H}$ (high). The preferences among these values are conditional to the bandwidth. If the bandwidth is low $\left(B_{L}\right)$, a low resolution $\left(R_{L}\right)$ has higher preference (very high), but if the bandwidth is high $\left(B_{H}\right)$, a high resolution $\left(R_{H}\right)$ is prefered (very high). When the bandwidth is medium $\left(B_{m}\right)$, a low resolution image is prefered, but with less intensity (high).

Using weights $0.6,0.25$ and 0.15 for $\mathrm{B}, \mathrm{S}$ and $\mathrm{R}$, we can compute, ${ }^{8}$ for example, the global utility of the assignment $\left(S_{f u l l}, B_{M}, R_{H}\right)$ as $\Delta(0.6 \times$ $2+0.25 \times 4+0.15 \times 4)=2.8=(3,-0.2)$ which gives the term high.

\section{Conclusions}

In this paper, we have presented a new variant of CP-Nets, called Linguistic CP-Nets (LCP-Nets), combining features of UCP-Nets and TCP-Nets with the advantages of a fuzzy linguistic approach to discretize continuous domain variables, and to express the utilities of assignments to variables in conditional preference tables. LCP-Nets prove to be well suited for semantic SOA, as they allow users to effectively discretize continuous domain QoS with appropriate linguistic terms, and to express utility of assignments in a qualitative manner rather than an often contrived numerical one.

We have assumed for the time being that utilities are always expressed using the same linguistic term set, but this restriction could easily be removed by using a multigranular ${ }^{9}$ approach when computing the global utility function. Similarly, the assumption on the linguistic sets as being centered on 0.5 with terms being equidistant could also be removed using approaches to cope with unbalanced term sets. ${ }^{7}$

\section{References}

1. F. Boubekeur and L. Tamine-Lechani. Recherche d'information flexible basée CP-Nets. In Proc. Conference on Recherche d'Information et Applications (CORIA'06), pages 161-167, March 2006.

2. C. Boutilier, F. Bacchus, and R. I. Brafman. UCP-Networks: A directed graphical representation of conditional utilities. In Proc. of the Seventeenth Conference on Uncertainty in Artificial Intelligence, pages 56-64, 2001.

3. C. Boutilier, R. I. Brafman, C. Domshlak, H. H. Hoos, and D. Poole. CPnets: A tool for representing and reasoning with conditional Ceteris Paribus 
Preference Statements. Journal of Artificial Intelligence Research, 21:135191, 2004.

4. R. I. Brafman and C. Domshlak. Introducing variable importance tradeoffs into CP-nets. In Proc. of the Eighteenth Annual Conference on Uncertainty in Artificial Intelligence, pages 69-76, 2002.

5. R. Degani and G. Bortolan. The Problem of Linguistic Approximation in Clinical Decision Making. International Journal of Approximate Reasoning, 2:143-162, 1988.

6. M. Delgado, J.L. Verdegay, and M.A Vila. On Aggregation Operations of Linguistic Labels. International Journal of Intelligent Systems, 8:351-370, 1993.

7. F. Herrera, E. Herrera-Viedma, and L. Martínez. A Hierarchical Ordinal Model for Managing Unbalanced Linguistic Term Sets Based on the Linguistic 2-Tuple Model. In EUROFUSE Workshop on Preference Modelling and Applications, pages 201-206, 2001.

8. F. Herrera and L. Martínez. A 2-tuple fuzzy linguistic representation model for computing with words. IEEE Transactions on Fuzzy Systems, 8(6):746$752,2000$.

9. F. Herrera, L. Martínez, E. Herrera-Viedma, and F. Chiclana. Fusion of Multigranular Linguistic Information based on the 2-tuple Fuzzy Linguistic Representation Model. In Proceedings of IPMU 2002, pages 1155-1162, 2002.

10. H. Lausen and D. Innsbruck. Semantic Annotations for WSDL and XML Schema, 2007.

11. C. Schröpfer, M. Binshtok, S. E. Shimony, A. Dayan, R. Brafman, P. Offermann, and O. Holschke. Introducing preferences over NFPs into service selection in SOA. In Proc. Non Functional Properties and Service Level Agreements in Service Oriented Computing Workshop (NFPSLA-SOC'O7), 2007.

12. L.A. Zadeh. The Concept of a Linguistic Variable and Its Applications to Approximate Reasoning. Information Sciences, Part I, II, III, 8,8,9:199-249, 301-357, 43-80, 1975. 